TITLE:

\title{
Singular behavior of the macroscopic quantities in the free molecular gas
}

\section{$\operatorname{AUTHOR}(\mathrm{S})$ :}

Takata, Shigeru; Yoshida, Tatsuya; Noguchi, Takashi; Taguchi, Satoshi

\section{CITATION:}

Takata, Shigeru ...[et al]. Singular behavior of the macroscopic quantities in the free molecular gas. Physics of Fluids 2016, 28(2): 0022002.

\section{ISSUE DATE:}

2016-02

URL:

http://hdl.handle.net/2433/243841

\section{RIGHT:}

This article may be downloaded for personal use only. Any other use requires prior permission of the author and AIP Publishing. This article appeared in S. Takata et al., Physics of Fluids 28, 022002 (2016), and may be found at https://doi.org/10.1063/1.4940988. 


\section{Singular behavior of the macroscopic quantities in the free molecular gas}

Cite as: Phys. Fluids 28, 022002 (2016); https://doi.org/10.1063/1.4940988

Submitted: 20 October 2015 . Accepted: 17 January 2016 . Published Online: 04 February 2016

Shigeru Takata (D), Tatsuya Yoshida, Takashi Noguchi, and Satoshi Taguchi (D)
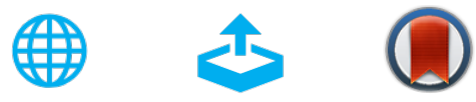

View Online

Export Citation

CrossMark

\section{ARTICLES YOU MAY BE INTERESTED IN}

Slip/jump coefficients and Knudsen-layer corrections for the ES model in the generalized slip-flow theory

AIP Conference Proceedings 1786, 040004 (2016); https://

doi.org/10.1063/1.4967542

Theoretical description of the gaseous Knudsen layer in Couette flow based on the second-order constitutive and slip-jump models

Physics of Fluids 28, 012002 (2016); https://doi.org/10.1063/1.4938240

Investigation of rarefied gas flow in microchannels of non-uniform cross section Physics of Fluids 28, 022007 (2016); https://doi.org/10.1063/1.4942183

\section{CAPTURE WHAT'S POSSIBLE}

WITH OUR NEW PUBLISHING ACADEMY RESOURCES

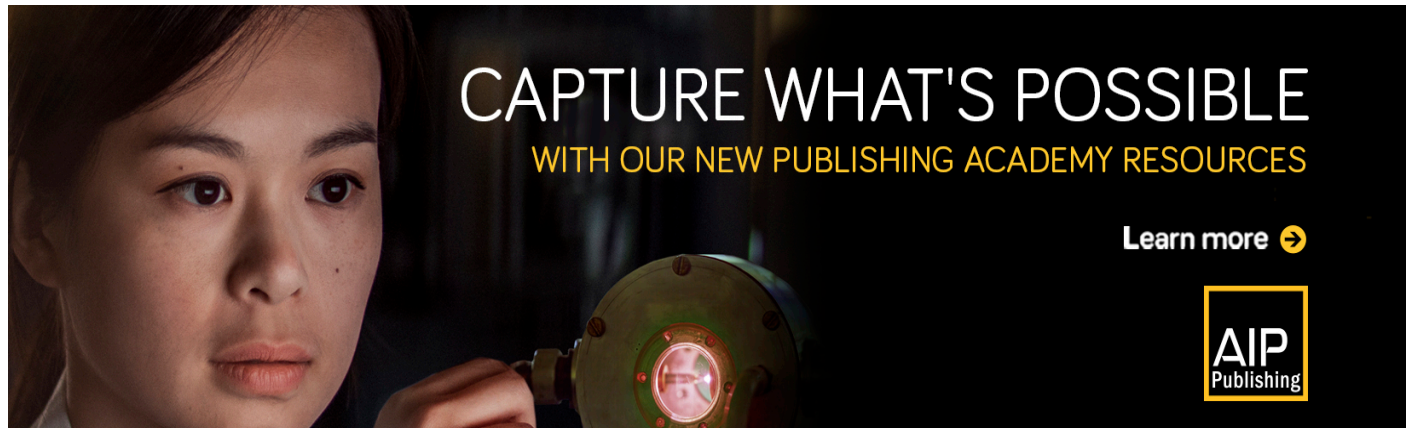




\title{
Singular behavior of the macroscopic quantities in the free molecular gas
}

\author{
Shigeru Takata, ${ }^{1, a)}$ Tatsuya Yoshida, ${ }^{1}$ Takashi Noguchi, ${ }^{1}$ \\ and Satoshi Taguchi ${ }^{2}$ \\ ${ }^{1}$ Department of Aeronautics and Astronautics, Kyoto University, Kyoto 615-8540, Japan \\ ${ }^{2}$ Department of Mechanical Engineering and Intelligent Systems, The University of \\ Electro-Communications, Chofu, Tokyo 182-8585, Japan
}

(Received 20 October 2015; accepted 17 January 2016; published online 4 February 2016)

\begin{abstract}
Steady behavior of the free molecular gas is studied with a special interest in the behavior around a convex body. Two types of singular behavior are shown to occur at the level of the macroscopic quantities. Their occurrence and the strength of singularity are discussed in detail both numerically and analytically. A universal law behind them is revealed by the consideration of the local geometry of the boundary. (C) 2016 AIP Publishing LLC. [http://dx.doi.org/10.1063/1.4940988]
\end{abstract}

\section{INTRODUCTION}

The conductivity or transport property in the usual fluid obeys Newton's stress law and Fourier's heat law. From the microscopic point of view, this is a consequence of frequent intermolecular collisions during the flight of each molecule. In a rarefied gas, however, such collisions become less frequent and the ballistic nature emerges in the conduction property. Accordingly, the fluid dynamical or macroscopic quantities of a rarefied gas exhibit a peculiar feature that is absent in the usual fluid. This is the topic that we would like to address in the present paper.

In the present paper, we single out the ballistic aspect of a rarefied gas by considering its collisionless limit. The gas in such a limit is called a free molecular gas. ${ }^{1}$ We will show that, due to the ballistic nature, the gradient of fluid dynamical or macroscopic quantities can diverge on the surface of a convex body in its normal direction, even if its temperature and geometry are smooth. Furthermore, we show that the gradient of macroscopic quantities may lose the smoothness or even has a jump discontinuity in the gas, so that their isolines show a sharp or "edged" bending. To our best knowledge, these (fluid-dynamically) strange features have not been mentioned explicitly or at least studied in detail so far. We do not simply show the occurrence of these features but rather clarify the mechanism of their occurrence. We reveal the universality behind them by the geometric argument. The clue is the propagation of discontinuity of the velocity distribution function, ${ }^{2}$ which reflects the ballistic transport and the (local) convexity of the body. The geometric classification thus naturally arises in our discussions.

The paper is organized as follows. The specific problem is introduced and formulated in Sec. II. The expression of the solution is derived by the use of a statics for the free molecular gas ${ }^{1,3,4}$ in Sec. III A. Then, a brief sketch of the way of computations follows in Sec. III B. Numerical results are presented in Sec. IV. The detailed analyses and discussions on the numerical results are given in Secs. IV A and IV B; the former is devoted to the study of the divergence on the body surface, while the latter to that of the sharp or edged bend in the isolines. The paper is concluded with Sec. V.

\footnotetext{
a) Also at Research Project of Fluid Science and Engineering, Advanced Engineering Research Center, Kyoto University, Kyoto 615-8540, Japan. Electronic mail: takata.shigeru.4a@kyoto-u.ac.jp
} 


\section{PROBLEM AND FORMULATION}

Consider a free molecular gas in the domain $D$ between two straight pipes with different cross sections, see Fig. 1. The inner pipe has a smooth convex cross section and is maintained at surface temperature $T_{w}$. Here, $T_{w}$ is nonuniform in general. The outer pipe has a rectangular cross section with dimensions $2 d_{1} \times 2 d_{2}$ and is maintained at uniform temperature $T_{0}$. There is no external force. We will investigate the steady behavior of the gas under the following assumptions:

1. The behavior of the gas is described by the collisionless Boltzmann equation for a monatomic gas.

2. The gas molecules are diffusely reflected ${ }^{1}$ on the inner and outer pipes.

3. The surface temperature of the inner pipe $T_{w}$ is uniform in the axial direction.

Let us denote by $a$ the characteristic length of the inner pipe cross section and introduce the Cartesian coordinates $X_{i}$ or $a x_{i}(i=1,2,3)$ with its origin at the center of the outer pipe cross section; see Fig. 1 . We set $X_{3}$ or $a x_{3}$ to be in the axial direction. Let us denote by $\left(2 R T_{0}\right)^{1 / 2} \zeta_{i}$ the molecular velocity and by $\rho_{s}\left(2 R T_{0}\right)^{-3 / 2} \phi\left(x_{1}, x_{2}, \zeta\right)$ the velocity distribution function of gas molecules. Here, $R$ is the specific gas constant and $\rho_{s}$ is the density of the gas in the equilibrium state at rest when the inner pipe is maintained at the uniform temperature $T_{0}$. Then, the problem is reduced to solving the following boundary-value problem:

$$
\begin{gathered}
\zeta_{1} \frac{\partial \phi}{\partial x_{1}}+\zeta_{2} \frac{\partial \phi}{\partial x_{2}}=0,(a x \in D), \\
\phi=\frac{\hat{\sigma}_{0}}{\pi^{3 / 2}} \exp \left(-\zeta_{j}^{2}\right),\left(\zeta_{j} n_{j}>0, G_{\text {out }}=0\right), \\
\hat{\sigma}_{0}=-2 \sqrt{\pi} \int_{\zeta_{j} n_{j}<0} \zeta_{j} n_{j} \phi d \zeta, \\
\phi=\frac{\hat{\sigma}_{w}}{\left(\pi \hat{T}_{w}\right)^{3 / 2}} \exp \left(-\frac{\zeta_{j}^{2}}{\hat{T}_{w}}\right),\left(\zeta_{j} n_{j}>0, G_{\text {in }}=0\right), \\
\hat{\sigma}_{w}=-2 \sqrt{\frac{\pi}{\hat{T}_{w}}} \int_{\zeta_{j} n_{j}<0} \zeta_{j} n_{j} \phi d \zeta,
\end{gathered}
$$

where $\hat{T}_{w}=T_{w} / T_{0}$ and $n_{j}$ is the unit vector normal to the inner $G_{i n}\left(x_{1}, x_{2}\right)=0$ or the outer surface $G_{\text {out }}\left(x_{1}, x_{2}\right)=0$, pointing to the gas side. The functional form of $G_{i n}$ will be specified soon later, while $G_{\text {out }}=\left(\left|x_{1}\right|-\hat{d}_{1}\right)\left(\left|x_{2}\right|-\hat{d}_{2}\right)$ for $\left|x_{1}\right| \leq \hat{d}_{1}$ and $\left|x_{2}\right| \leq \hat{d}_{2}$, where $\hat{d}_{i}=d_{i} / a(i=1,2)$. In what follows, we simply call the pipe surfaces the boundary and follow the convention that the curvature of the boundary, $\kappa$, is positive when the center of curvature does not lie in the gas side.

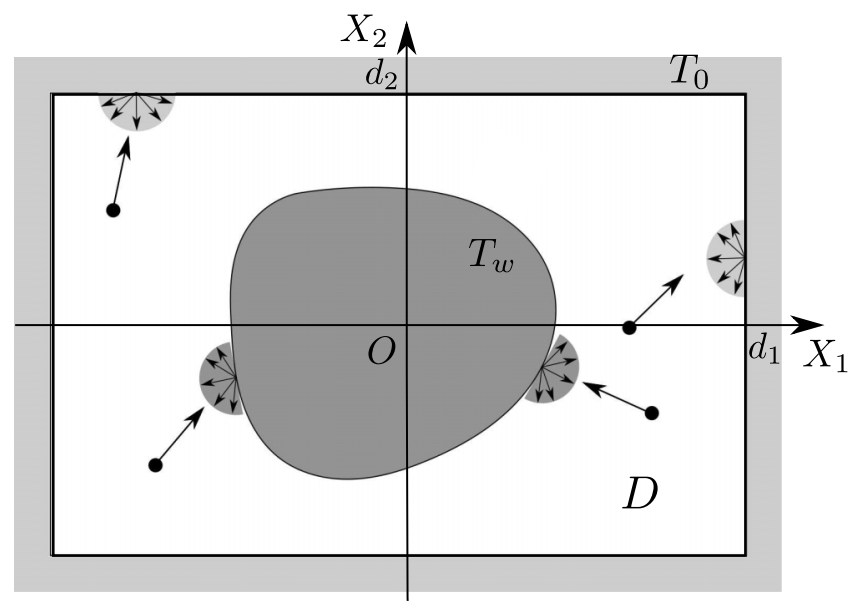

FIG. 1. Problem configuration. 


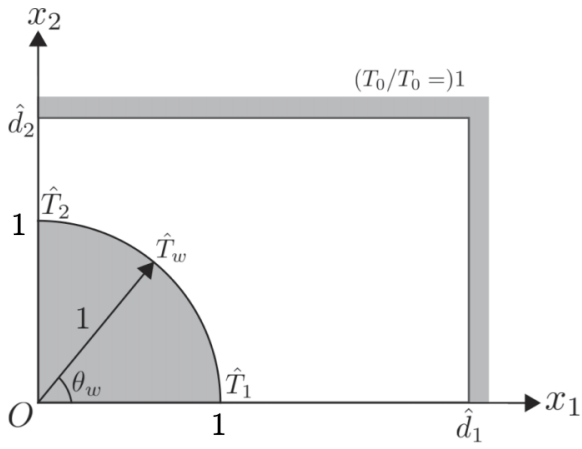

(a) G1

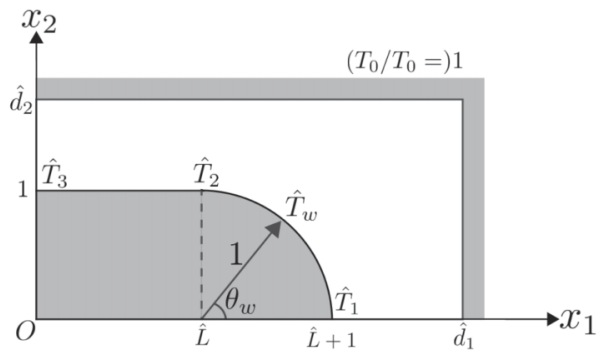

(c) G3

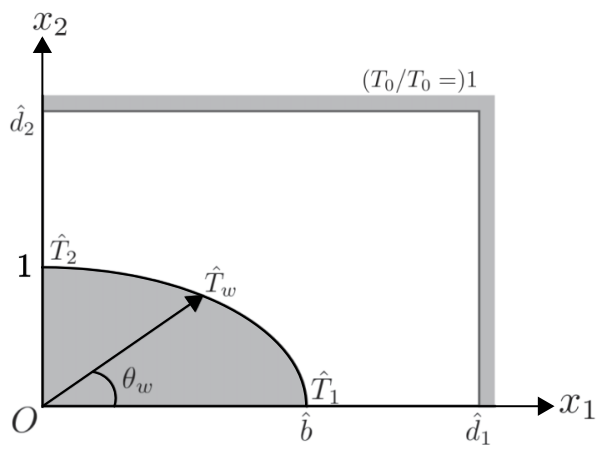

(b) G2

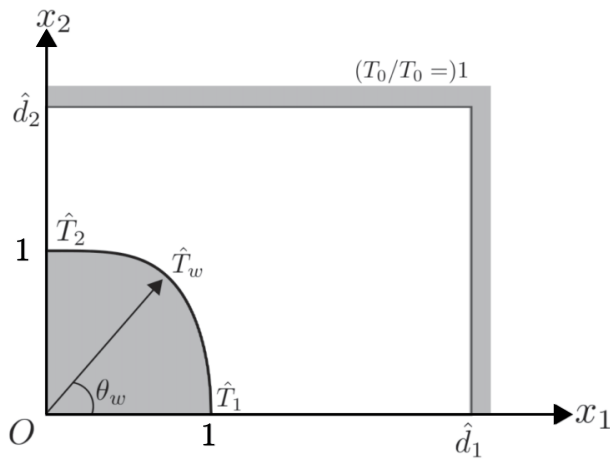

(d) G4

FIG. 2. Four types of setting: (a) G1, (b) G2, (c) G3, and (d) G4. Only the first quadrant is shown.

The gas density $\rho_{s} \hat{\rho}$, temperature $T_{0} \hat{T}$, flow velocity $\left(2 R T_{0}\right)^{1 / 2} \hat{v}_{i}$, and heat-flow vector $\rho_{s} R T_{0}\left(2 R T_{0}\right)^{1 / 2} \hat{q}_{i}$ are given by the following moments of $\phi$ :

$\hat{\rho}=\int \phi d \zeta, \quad \hat{v}_{i}=\frac{1}{\hat{\rho}} \int \zeta_{i} \phi d \zeta, \quad \hat{T}=\frac{2}{3 \hat{\rho}} \int\left(\zeta_{i}-\hat{v}_{i}\right)^{2} \phi d \zeta, \quad \hat{q}_{i}=\int\left(\zeta_{i}-\hat{v}_{i}\right)\left(\zeta_{j}-\hat{v}_{j}\right)^{2} \phi d \zeta$.

We will consider four types of physical setting (G1-G4) (Fig. 2), G1:

$$
\begin{aligned}
& G_{i n}\left(x_{1}, x_{2}\right)=x_{1}^{2}+x_{2}^{2}-1, \\
& \hat{T}_{w}=\hat{T}_{1}+\left(\hat{T}_{2}-\hat{T}_{1}\right)\left|\sin \theta_{w}\right|, \quad\left(-\pi<\theta_{w} \leq \pi\right) .
\end{aligned}
$$

G2:

$$
\begin{aligned}
& G_{i n}\left(x_{1}, x_{2}\right)=x_{1}^{2} / \hat{b}^{2}+x_{2}^{2}-1, \\
& \hat{T}_{w}=\hat{T}_{1}+\left(\hat{T}_{2}-\hat{T}_{1}\right) \sin ^{2} \theta_{w}, \quad\left(-\pi<\theta_{w} \leq \pi\right) .
\end{aligned}
$$

G3:

$$
\begin{aligned}
& G_{i n}\left(x_{1}, x_{2}\right)= \begin{cases}\left|x_{2}\right|-1, & \left(-\hat{L} \leq x_{1} \leq \hat{L}\right), \\
\left(\left|x_{1}\right|-\hat{L}\right)^{2}+x_{2}^{2}-1, & \left(\hat{L}<\left|x_{1}\right| \leq \hat{L}+1\right),\end{cases} \\
& \hat{T}_{w}= \begin{cases}\hat{T}_{2}+\left(\hat{T}_{3}-\hat{T}_{2}\right) \cos ^{2}\left(\frac{\pi}{2} \frac{x_{1}}{\hat{L}}\right), & \left(-\hat{L} \leq x_{1} \leq \hat{L}\right), \\
\hat{T}_{1}+\left(\hat{T}_{2}-\hat{T}_{1}\right) \sin ^{2} \theta_{w}, & \left(-\pi<\theta_{w} \leq \pi\right) .\end{cases}
\end{aligned}
$$

G4:

$$
\begin{aligned}
& G_{i n}\left(x_{1}, x_{2}\right)=x_{1}^{4}+x_{2}^{2}-1, \\
& \hat{T}_{w}=\hat{T}_{2}+\left(\hat{T}_{1}-\hat{T}_{2}\right) \sin ^{2}\left(\frac{\pi}{2} x_{1}\right), \quad\left(-1 \leq x_{1} \leq 1\right) .
\end{aligned}
$$


Here $\hat{T}_{1}, \hat{T}_{2}$, and $\hat{T}_{3}$ are parameters for the temperature distribution of the inner boundary, $\hat{b}$ and $\hat{L}$ are parameters for its geometry, and $\theta_{w}$ is the polar angle specifying the point on the inner boundary shown in Fig. 2. The important feature of the inner boundary geometry $G_{i n}=0$ is as follows. In $\mathrm{G} 1$, the curvature $\kappa$ is positive and common to all the points of the inner boundary. In G2, $\kappa$ is positive but dependent on the boundary position. In G3, there is a flat part $(\kappa=0)$ and a circular arc part $(\kappa>0)$. In $\mathrm{G} 4$, on the whole surface $\kappa>0$, except for the point $\left(x_{1}, x_{2}\right)=(0,1)$. At this point, $\kappa=0$, though the boundary is not flat. Furthermore, it should be noted that there are a few exceptional points in the following sense of smoothness: (i) $\hat{T}_{w}$ is not smooth at $x_{1}= \pm 1$ in $\mathrm{G} 1$. (ii) The curvature of boundary is not continuous at $x_{1}= \pm \hat{L}$ in G3. The second derivative of the temperature along the surface is not necessarily continuous there in G3.

Because both of the boundary geometry and the temperature distribution $\hat{T}_{w}$ are symmetric with respect to the $x_{1}$-axis and $x_{2}$-axis, we will consider only the first quadrant, as shown in Fig. 2.

\section{OUTLINE OF THE SOLUTION METHOD}

\section{A. Application of statics for the free molecular gas}

The free molecular gas is the collisionless limit of the gas that models an extremely rarefied gas. In such a limit, the gas molecules go straight if there is no external force. Accordingly, the characteristic of Eq. (1a) is a straight line, along which the velocity distribution function $\phi$ is constant. Therefore, it is straightforward to have a general solution of Eq. (1a) itself. Nevertheless, it is not trivial to find a solution of the boundary-value problem (1), because $\hat{\sigma}_{0}$ and $\hat{\sigma}_{w}$ in the boundary data (1b) and (1d) for reflected molecules are related to the flux of molecules impinging on the boundary through Eqs. (1c) and (1e).

Fortunately, however, we can make use of a general solution algorithm established in Refs. 3 and 4 in the present problem. For the later convenience, let us introduce the polar coordinates $\left(\zeta_{\rho}, \psi\right)$ for the components of the molecular velocity $\left(\zeta_{1}, \zeta_{2}\right)$ in the plane of the cross section, i.e., $\zeta_{1}=\zeta_{\rho} \cos \psi$ and $\zeta_{2}=\zeta_{\rho} \sin \psi\left(0 \leq \zeta_{\rho}<\infty,-\pi<\psi \leq \pi\right)$. Then, the solution of the present problem (1) is expressed as

$$
\phi\left(x_{1}, x_{2}, \zeta\right)= \begin{cases}\frac{\pi^{-3 / 2}}{\hat{T}_{w}^{2}\left(x_{1}^{B}, x_{2}^{B}\right)} \exp \left(-\frac{\zeta_{\rho}^{2}+\zeta_{3}^{2}}{\hat{T}_{w}\left(x_{1}^{B}, x_{2}^{B}\right)}\right), & \text { for } \psi_{1} \leq \psi \leq \psi_{2}, \\ \pi^{-3 / 2} \exp \left(-\zeta_{\rho}^{2}-\zeta_{3}^{2}\right), & \text { otherwise, }\end{cases}
$$

where $\psi_{1}$ and $\psi_{2}$ are the polar angles of the tangential lines from the point $\left(x_{1}, x_{2}\right)$ to the inner boundary $\left(-\pi<\psi_{1}<\psi_{2} \leq \pi\right)$, thus depending on $\left(x_{1}, x_{2}\right)$. The position $\left(x_{1}^{B}, x_{2}^{B}\right)$ is the intersection of the inner boundary with the straight line through the point $\left(x_{1}, x_{2}\right)$ with the slope $\tan \psi$ (see Fig. 3). As is seen from Eq. (7), $\phi$ is independent of $\hat{d}_{1}$ and $\hat{d}_{2}$; thus, we do not specify their values.

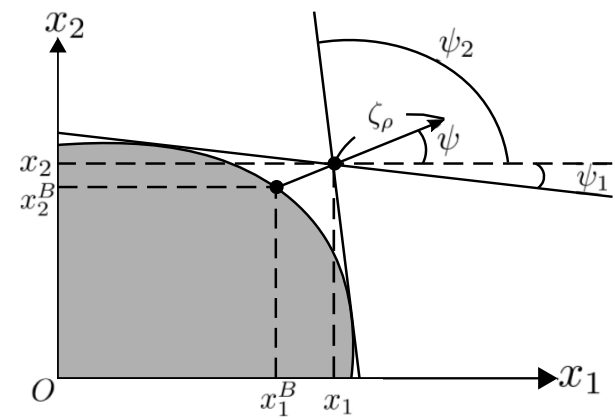

FIG. 3. Polar angles $\psi_{1}$ and $\psi_{2}$ of the tangential lines, and the intersection $\left(x_{1}^{B}, x_{2}^{B}\right)$ of the surface with the characteristic line. 
Substituting Eq. (7) in Eq. (2) yields the following expressions for $\hat{\rho}, \hat{v}_{i}, \hat{T}$, and $\hat{q}_{i}$ :

$$
\begin{aligned}
& \hat{\rho}=\frac{1}{2 \pi}\left(2 \pi+\int_{\psi_{1}}^{\psi_{2}}\left(\hat{T}_{w}^{-1 / 2}\left(x_{1}^{B}, x_{2}^{B}\right)-1\right) d \psi\right), \\
& \hat{v}_{i}=0, \\
& \hat{T}=\frac{1}{2 \pi \hat{\rho}}\left(2 \pi+\int_{\psi_{1}}^{\psi_{2}}\left(\hat{T}_{w}^{1 / 2}\left(x_{1}^{B}, x_{2}^{B}\right)-1\right) d \psi\right), \\
& \hat{q}_{1}=\frac{1}{2 \sqrt{\pi}} \int_{\psi_{1}}^{\psi_{2}}\left(\hat{T}_{w}\left(x_{1}^{B}, x_{2}^{B}\right)-1\right) \cos \psi d \psi, \\
& \hat{q}_{2}=\frac{1}{2 \sqrt{\pi}} \int_{\psi_{1}}^{\psi_{2}}\left(\hat{T}_{w}\left(x_{1}^{B}, x_{2}^{B}\right)-1\right) \sin \psi d \psi, \\
& \hat{q}_{3}=0,
\end{aligned}
$$

among which we will be mainly concerned with $\hat{\rho}$ and $\hat{T}$.

It is seen from Eq. (8b) that the steady flow is not induced in the present problem. It is not trivial, because, in contrast to the usual fluid, various steady flows are known to be induced thermally in a rarefied gas, ${ }^{5,6}$ such as the thermal creep,,${ }^{5,7-9}$ thermal stress-slip, ${ }^{10,11}$ nonlinear thermal-stress, ${ }^{12}$ and thermal-edge flows. ${ }^{13,14}$ Thermally driven compressors and gas separators were also proposed and studied in the last two decades. ${ }^{15-19}$ No occurrence of flow in the free molecular limit is one of the remarkable general statements of the statics (see Ref. 1), which applies to a wide class of problems, assuming the Maxwell's boundary condition. Incidentally, if another type of boundary condition such as the Cercignani-Lampis condition ${ }^{20}$ is assumed, the thermally induced flow remains finite even in the free molecular limit. ${ }^{21}$ However, the magnitude of the remaining flow is small, compared to that for finite Knudsen numbers. Detailed discussions can be found in Ref. 21.

\section{B. Sketch of actual computations}

The computations of the macroscopic quantities at each spatial position $\left(x_{1}, x_{2}\right)$ through Eq. (8) are essentially reduced to those of the polar angles $\psi_{1}$ and $\psi_{2}$ and the intersection point $\left(x_{1}^{B}, x_{2}^{B}\right)$; see Fig. 3. For every fixed $\psi$, the intersection is determined by finding a solution of the simultaneous equations

$$
\begin{aligned}
& x_{2}^{B}=\left(x_{1}^{B}-x_{1}\right) \tan \psi+x_{2}, \\
& G_{i n}\left(x_{1}^{B}, x_{2}^{B}\right)=0,
\end{aligned}
$$

which minimizes $\left(x_{1}-x_{1}^{B}\right)^{2}+\left(x_{2}-x_{2}^{B}\right)^{2}$. The polar angles $\psi_{1}$ and $\psi_{2}$ are determined to be the angle for which Eq. (9) has a multiple root.

In the case of G1-G3, Eq. (9) is reduced to at most the second-order algebraic equation, so that $\psi_{1}$ and $\psi_{2}$ [or $\left(x_{1}^{B}, x_{2}^{B}\right)$ ] can be written explicitly as functions of $\left(x_{1}, x_{2}\right)$ [or $\left(x_{1}, x_{2}\right)$ and $\psi$ ]. In the case of G4, Eq. (9) is the fourth-order algebraic equation, and we compute $\psi_{1}, \psi_{2}$, and $\left(x_{1}^{B}, x_{2}^{B}\right)$ numerically by the Newton-Raphson method. ${ }^{23}$ Although the functional forms of the integrands in Eq. (8) are known for G1-G3, we commonly apply the composite Simpson rule to the numerical integrations in Eq. (8) for G1-G4.

\section{RESULTS AND DISCUSSIONS}

We carried out the computations for various values of $\hat{T}_{1}, \hat{T}_{2}$, and $\hat{T}_{3}$ for each G1-G4. Representative results are shown in Figs. 4-7. In each figure, the panel (a) shows the isoline map of the temperature $\hat{T}$ and the vector field of the heat flow $\hat{q}_{i}$, while the panel (b) the isoline map of the density $\hat{\rho}$.

In Figs. 4-7, the heat flow is commonly induced from the hotter to the colder region, which is the same tendency as in the usual fluid. However, it does not imply that Fourier's heat law holds in the present problem. As is seen from Eq. (7), the transport property of the free molecular 


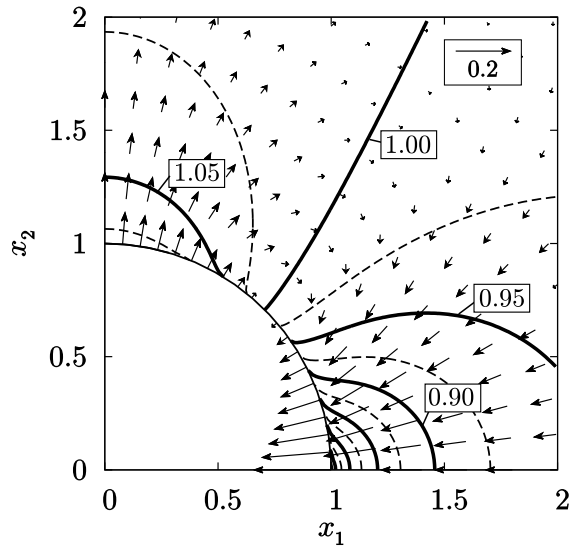

(a)

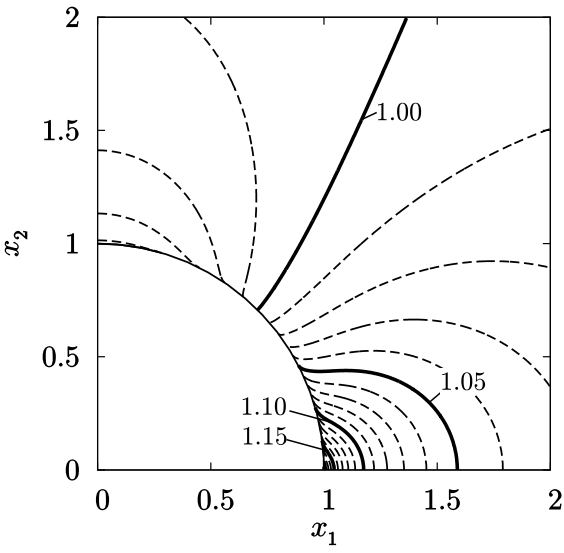

(b)

FIG. 4. Temperature, heat-flow, and density fields for G1 $\left[\hat{T}_{1}=1 / 2\right.$ and $\left.\hat{T}_{2}=(1+\sqrt{2}) / 2\right]$. (a) The isoline map of $\hat{T}$ and vector field of $\hat{q}_{i}$. (b) The isoline map of $\hat{\rho}$. In panel (a), the arrows indicate the heat-flow vector $\hat{q}_{i}$ at their starting points. The scale of the vector is shown on the top right in the panel.

gas is ballistic and the resulting temperature field is necessarily different from that described by the heat-conduction equation for the usual resting fluid. For instance, the jump in temperature is observed between the gas and the boundary in the figures, which would be checked more easily on the outer pipe because its (dimensionless) temperature is unity. It is one of the typical phenomena in a rarefied gas, known as the temperature jump on the boundary. $1,5,24$

The isoline maps of the density in Figs. 4-7 look similar to those of the temperature, though their increasing/decreasing trends are opposite to each other. In the case of G3 and G4, the isolines of both temperature and density look sharply bent on the line $x_{2}=1$ in the gas; see Figs. 6 and 7 . Such a strange feature is not observed in G1 and G2; see Figs. 4 and 5.

In Secs. IV A and IV B, we will discuss in detail the behavior of the macroscopic quantities near the inner boundary and the strange feature of isolines observed in G3 and G4. The discussions in Secs. IV A and IV B are the core of the present paper.

\section{A. Universality of the gas behavior near the convex boundary}

Let us denote by $s$ the normal distance from the inner boundary (see Fig. 8 in the case of G1). Figure 9 shows the variation of the density $\hat{\rho}$ near the inner boundary as a function of $s$. The panels (a)-(d) in the figure correspond to the cases shown in panel (b) of Figs. 4-7. Several points on the boundary are chosen and specified by the polar angle $\theta_{w s}$ or coordinate $x_{s}$ illustrated in Fig. 8. It is found that the variation obeys the 1/2-power law in the vicinity most of those points. This suggests

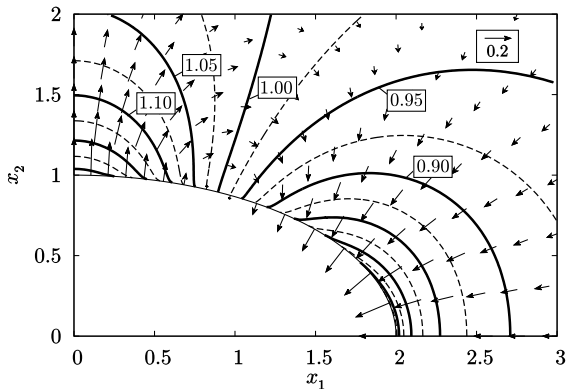

(a)

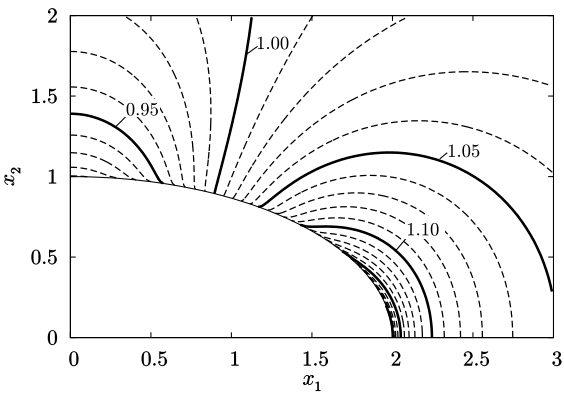

(b)

FIG. 5. Temperature, heat-flow, and density fields for $\mathrm{G} 2\left(\hat{b}=2, \hat{T}_{1}=1 / 2, \hat{T}_{2}=3 / 2\right)$. See the caption of Fig. 4. 


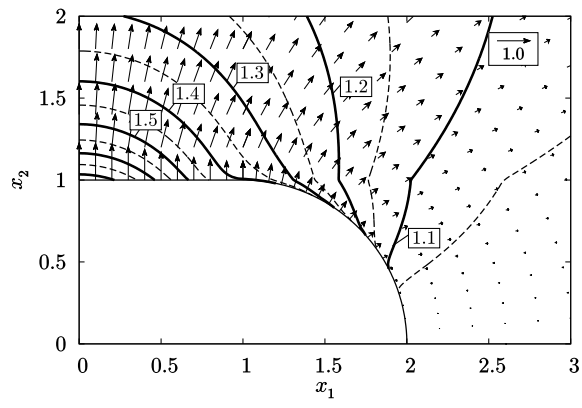

(a)

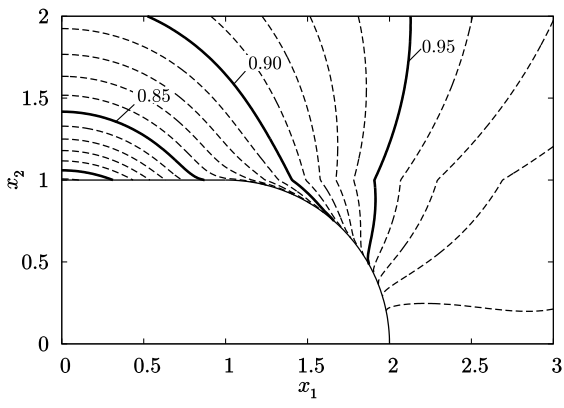

(b)

FIG. 6. Temperature, heat-flow, and density fields for G3 $\left(\hat{L}=1, \hat{T}_{1}=1, \hat{T}_{2}=2, \hat{T}_{3}=3\right)$. See the caption of Fig. 4 .

the existence of universality for the behavior near the boundary: the gradient of $\hat{\rho}$ in the direction normal to the boundary diverges with the rate $s^{-1 / 2}$ as $s \rightarrow 0$. However, the different behavior is also observed at $\theta_{w s}=\pi / 4$ in G1 and G2, at the flat part of the boundary in G3 $\left(0 \leq x_{s}<\hat{L}\right)$, and at $\theta_{w s}=0$ and $\theta_{w s}=\pi / 2$ in G4. In these cases except for the last, the gradient of density remains finite. In the last case, i.e., at $\theta_{w s}=\pi / 2$ in $\mathrm{G} 4$, the gradient diverges with the weaker rate $s^{-1 / 4}$ as $s \rightarrow 0$.

Keeping in mind the above observations, let us first discuss the 1/2-power law in G1, which is geometrically simplest. We denote the derivative with respect to $s$ by . Below, the quantities dependent on $\left(x_{1}, x_{2}\right)$, such as $\psi_{1}, \psi_{2}$, and $\hat{T}_{w}$ in Eq. (8), should be understood as functions of $\left(\theta_{w s}, s\right)$ or $\left(x_{s}, s\right)$. Note that dependence of $\hat{T}_{w}$ on $s$ occurs through its dependence on $\theta_{w}$. Then, taking the derivative of Eq. (8a) with respect to $s$ yields

$$
\hat{\rho}^{\prime}=\frac{1}{2 \pi}\left[F\left(\cdot, \cdot, \psi_{2}\right) \psi_{2}^{\prime}-F\left(\cdot, \cdot, \psi_{1}\right) \psi_{1}^{\prime}\right]+\frac{1}{2 \pi} \int_{\psi_{1}}^{\psi_{2}} F^{\prime} d \psi
$$

where $F$ is the integrand in Eq. (8a), i.e.,

$$
F\left(\theta_{w s}, s, \psi\right)=\hat{T}_{w}\left(\theta_{w}\left(\theta_{w s}, s, \psi\right)\right)^{-1 / 2}-1,
$$

and $\psi_{1}, \psi_{2}$, and $\theta_{w}$ are expressed as functions of $s$ through the relations that

$$
\begin{aligned}
& (s+1) \sin \left(\psi-\theta_{w s}\right)=\sin \left(\psi-\theta_{w}\right), \\
& -\sin \left(\psi_{1}-\theta_{w s}\right)=\sin \left(\psi_{2}-\theta_{w s}\right)=1 /(s+1) .
\end{aligned}
$$

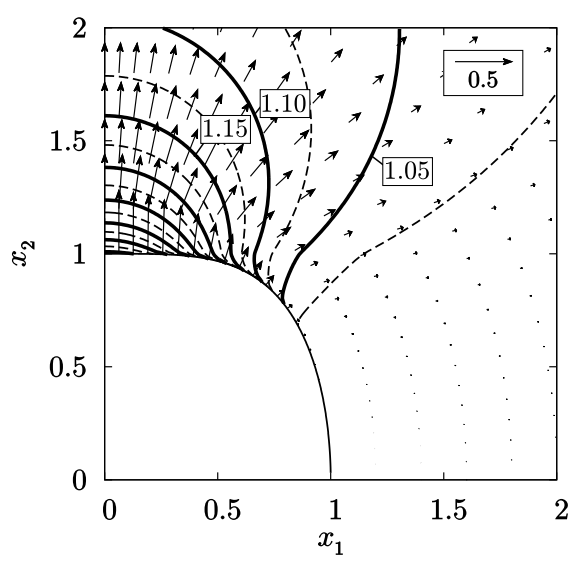

(a)

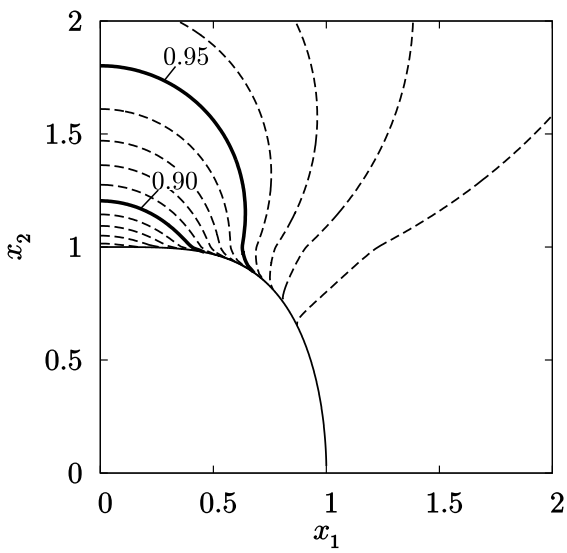

(b)

FIG. 7. Temperature, heat-flow, and density fields for G4 $\left(\hat{T}_{1}=1, \hat{T}_{2}=2\right)$. See the caption of Fig. 4. 


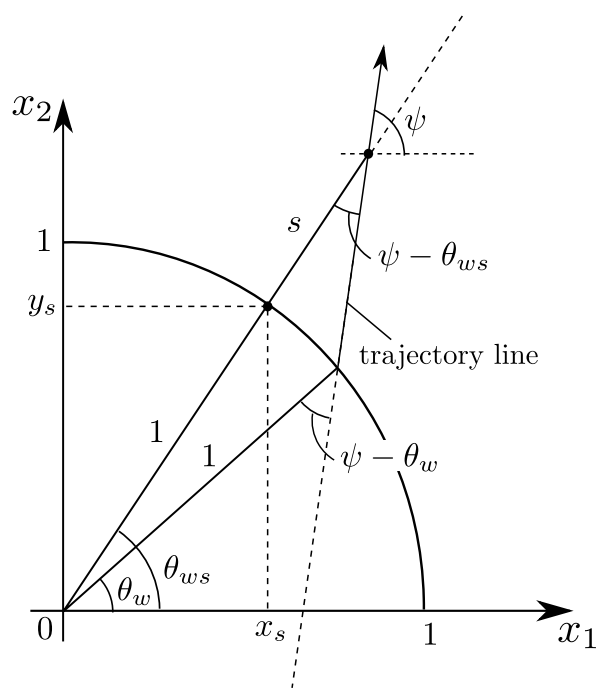

FIG. 8. Distance $s$ of the point $\left(x_{1}, x_{2}\right)$ from the boundary in the case of G1. The nearest boundary point is specified with the polar angle $\theta_{w s}$ or the coordinates $\left(x_{s}, y_{s}\right)$.

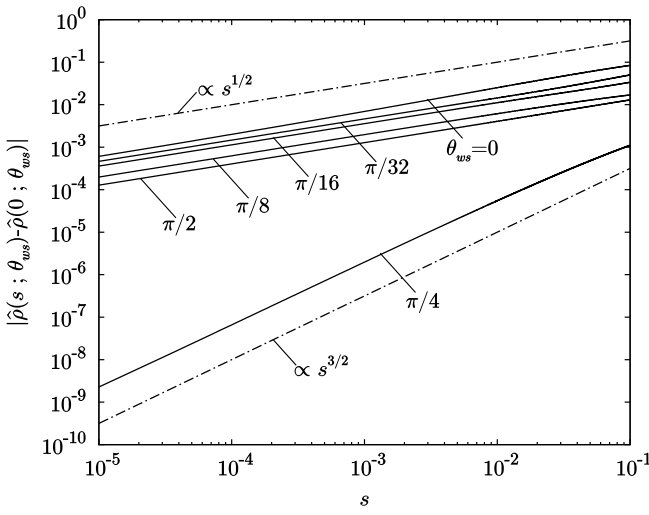

(a) G1

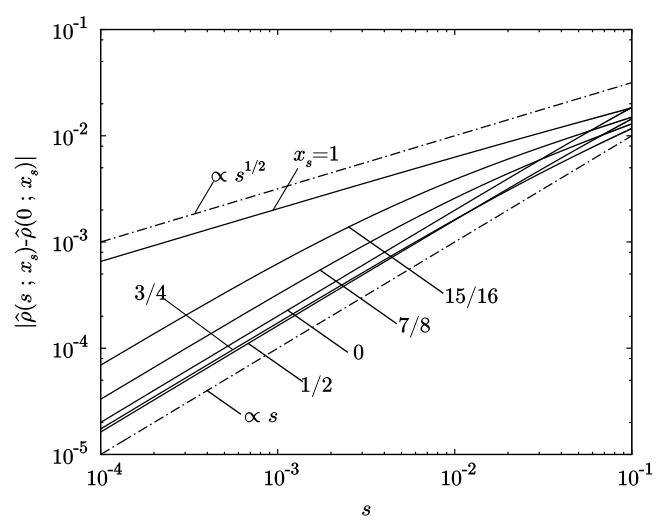

(c) G3

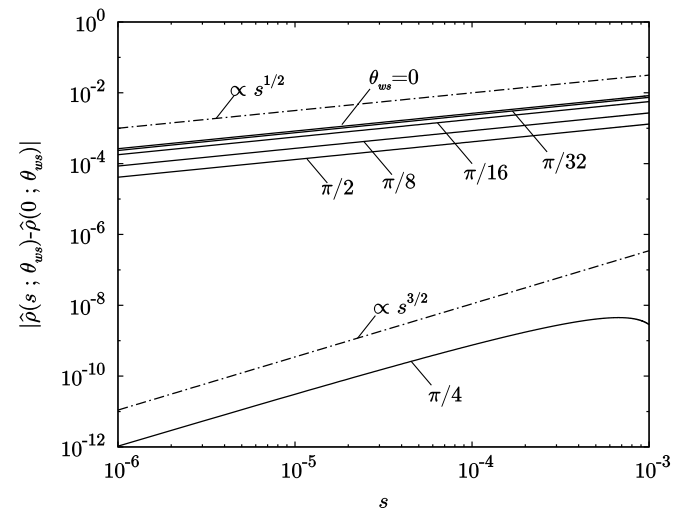

(b) G2

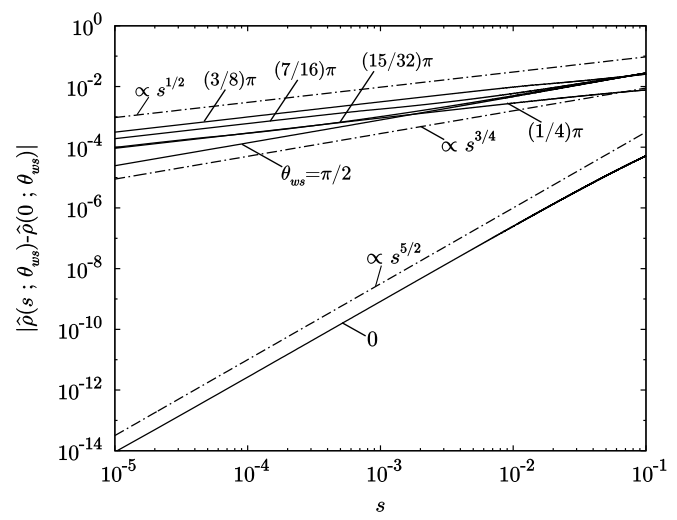

(d) G4

FIG. 9. Density variation from the boundary in its normal direction. (a) G1 $\left[\hat{T}_{1}=1 / 2, \hat{T}_{2}=(1+\sqrt{2}) / 2\right]$. (b) G2 $(\hat{b}=2$, $\left.\hat{T}_{1}=1 / 2, \hat{T}_{2}=3 / 2\right)$. (c) G3 $\left(\hat{L}=1, \hat{T}_{1}=1, \hat{T}_{2}=2, \hat{T}_{3}=3\right)$. (d) G4 $\left(\hat{T}_{1}=1, \hat{T}_{2}=2\right)$. 
Here, Eq. (11b) is a simple geometric consequence (see Fig. 8), while Eq. (11c) is its special (two) cases where the trajectory line in the figure is tangential to the inner boundary. Note that the $s$-derivative of the polar angles of tangential lines, $\psi_{1}^{\prime}$ and $\psi_{2}^{\prime}$, appear in the first term on the right-hand side of Eq. (10). This reflects the fact that the region of integration in Eq. (8a) changes according to the distance $s$ from the boundary, due to the discontinuity of $\phi$ at the directions $\psi=\psi_{1}$ and $\psi_{2}$; see Eq. (7). The second term in Eq. (10) is the integral of the $s$-derivative of the integrand of Eq. (8a). As far as the temperature $\hat{T}_{w}$ (and thus $F$ ) is smooth in $\theta_{w}$, the second term vanishes as $s \rightarrow 0$. The reader is referred to the Appendix for the proof and related discussions. Thus, the first term in Eq. (10), i.e., the change of the polar angle of the tangential line, should be the source of the power law of our concern. Indeed, it is easily seen from Eq. (11c) that

$$
\psi_{1}^{\prime}=-\psi_{2}^{\prime}=\frac{1}{(s+1) \sqrt{s(s+2)}} .
$$

Both $\psi_{1}^{\prime}$ and $\psi_{2}^{\prime}$ diverge with the rate $s^{-1 / 2}$ as $s \rightarrow 0$, so does the first term of Eq. (10). This explains the reason why the variation of $\hat{\rho}$ near the boundary obeys the $1 / 2$-power law in $s$. It should be reminded that Eq. (12) purely comes from the geometric property of the boundary.

Since any boundary with non-zero curvature is locally identical to a circular arc, the discussions in the previous paragraph apply to the vicinity of any boundary point with a positive curvature. In this way, we see that the 1/2-power law should apply to the inner boundary entirely in G1 and G2, its circular arc part in G3, and the entire inner boundary except for $\theta_{w s}=\pi / 2$ in G4. The $1 / 2$-power law is concluded to be a curvature effect.

There are still, however, some points with nonzero curvature at which the 1/2-power law does not hold: the points at $\theta_{w s}=\pi / 4$ in G1 and G2 and at $\theta_{w s}=0$ in G4. The clue to understand these exceptions is the fact that $\hat{T}_{w}$ at those points is commonly equal to unity by chance, i.e., the same temperature as the outer boundary. In case of such a coincidence in temperature, $F\left(\cdot, \cdot, \psi_{1}\right)$ and $F\left(\cdot, \cdot, \psi_{2}\right)$ are close to each other to make the singular factor in Eq. (12) less effective as $s \rightarrow 0$. Indeed, because $\theta_{w}\left(\theta_{w s}, 0, \psi\right)=\theta_{w s}, \hat{T}_{w}$ in Eq. (11a) can be estimated for $s \ll 1$ as

$$
\begin{aligned}
& \hat{T}_{w}\left(\theta_{w}\right)=\hat{T}_{w}\left(\theta_{w s}\right)+\left.\frac{d \hat{T}_{w}}{d \theta_{w}}\right|_{\theta_{w}=\theta_{w s}} \Theta+O\left(\Theta^{2}\right), \\
& \Theta \equiv \theta_{w}-\theta_{w s},
\end{aligned}
$$

where $\Theta$ is estimated at $\psi=\psi_{1}$ and $\psi=\psi_{2}$ as

$$
\Theta\left(\theta_{w s}, s, \psi\right)= \begin{cases}(2 s)^{1 / 2}+O\left(s^{3 / 2}\right), & \text { when } \psi=\psi_{1}, \\ -(2 s)^{1 / 2}+O\left(s^{3 / 2}\right), & \text { when } \psi=\psi_{2} .\end{cases}
$$

Since $\hat{T}_{w}\left(\theta_{w s}\right)=1$, the magnitude of $F\left(\cdot, \cdot, \psi_{1}\right)$ and $F\left(\cdot, \cdot, \psi_{2}\right)$ in Eq. (10) is $O\left(s^{1 / 2}\right)$, not $O(1)$. Furthermore, they cancel each other out at this order, leaving the contributions of $O(s)$ at most. Consequently, the first term of Eq. (10) as a whole degenerates into $O\left(s^{1 / 2}\right)$, the same order as that of the second term (see the Appendix). A further cancellation may happen, depending on the distribution of the surface temperature $\hat{T}_{w}$. In this way, both terms in Eq. (10) change moderately and the gradient of $\hat{\rho}$ does not diverge. The degeneracy of singularity is thus caused by vanishing the discontinuity of the velocity distribution function $\phi$ in the tangential direction to the boundary when approaching the boundary. Here the trigger of the vanishing discontinuity is the coincidence in temperature. In Figs. 9(a) and 9(b), $\hat{\rho}$ obeys the 3/2-power law, which supports the above explanation. In Fig. 9(d), $\hat{\rho}$ obeys the 5/2-power law, which is higher by one order than that at the other two points. The occurrence of the high order degeneracy is due to the very small variation of the surface temperature there.

An extension of the above discussions on the curvature effect is possible. Let us consider the boundary that is locally given by $z=x^{2 n}$, where $n$ is a natural number and $z<x^{2 n}$ is supposed to be the gas side. Then, it is easily seen that $\psi_{1}^{\prime}, \psi_{2}^{\prime} \propto[s /(2 n-1)]^{-1 / 2 n}$, and consequently the variation of $\hat{\rho}$ is proportional to $2 n[s /(2 n-1)]^{1-1 / 2 n}$ near the boundary. The point at $\theta_{w s}=\pi / 2$ in G4 is exactly the case $n=2$ and the density variation there shown in Fig. 9(d) obeys the 3/4-power law as predicted by the present extended argument. Note that $n=1$ is the boundary with nonzero 
curvature and we recover the 1/2-power law. The case $n \geq 2$ is the convex body without curvature. The limit $n \rightarrow \infty$ is the flat boundary and predicts the 1st-power law. Thus, the behavior on the flat part in G3 in Fig. 9(c) is also explained by the present extension. In this way, we have reached the conclusion that, even though the variation of the surface temperature is smooth, the normal gradient of the density diverges, in general, due to the local geometry of the boundary. We have also reached a comprehensive understanding on the universality of the power law for the normal gradient.

Although we have discussed so far only the density, the temperature also follows the same universality. Thus, the normal gradient of temperature also diverges at the position where that of the density diverges. It seems unreasonable from the viewpoint of the conventional fluid dynamics, because it suggests the infinite heat flux to the boundary. It is not the case, as is clearly observed in panel (a) in Figs. 4-7. Rather, the divergence of the normal gradient of temperature is the clear manifestation of the breakdown of Fourier's heat law in a rarefied gas. Even when there occur the intermolecular collisions, the divergence of the gradient of macroscopic quantities still occurs. Detailed studies on this issue can be found, for instance, in Ref. 22.

\section{B. A sharp and an edged bend of isoline}

In the present subsection, we study in detail the strange behavior of the isolines, in particular the isothermal lines, occurring in G3 and G4; see Figs. 6 and 7. The method and the results below apply also to the isoline of the density, though we omit its explanation here.

In viewing Figs. 6 and 7, the isolines look bent sharply or even with a sharp edge on the line $x_{2}=1$. This line is the tangential to the inner boundary at $\left(x_{1}, x_{2}\right)=(0,1)$, where the curvature of the boundary is zero both in G3 and G4. If the isothermal line is really bent with a sharp edge at some point (namely, if it has an "edged bend" there), the gradient of temperature has a jump discontinuity there. In order to see whether it is the case, we show in Fig. 10 the profile of $d \hat{T} / d x_{2}$ around $x_{2}=1$ along the lines $x_{1}=2,3$, and 4 in panel (a) for G3 and along the lines $x_{1}=1,2$, and 3 in panel (b) for G4. In drawing the figure, we have used the expression of $d \hat{T} / d x_{2}$ obtained from Eqs. (8a) and (8c) by taking their $x_{2}$-derivative.

It is clearly seen that $d \hat{T} / d x_{2}$ has a jump discontinuity at $x_{2}=1$ in Fig. 10(a). Thus, the isothermal line is bent with a sharp edge in G3. On the other hand, a different feature is observed in Fig. 10(b). The gradient $d \hat{T} / d x_{2}$ is continuous but changes very steeply at $x_{2}=1$ in G4.

In order to study the latter feature more closely, we consider the case that the geometry of the inner boundary is generalized as $x_{1}^{2 n}+x_{2}^{2}=1$, where $n$ is a natural number, but the surface temperature $\hat{T}_{w}$ is uniform, say $\hat{T}_{c}(\neq 1)$, for simplicity. Numerical results for G4 with $\hat{T}_{1}=\hat{T}_{2}=2$ exhibit the same feature as that in Fig. 7; thus, the simplification of the temperature does not spoil the essence of the phenomenon of our interest; see Fig. 11. Without loss of generality, we may restrict our consideration in the first quadrant. Note that the boundary is a circle when $n=1$ and that $\mathrm{G} 4$ is recovered when $n=2$. The $x_{2}$-gradient of temperature $d \hat{T} / d x_{2}$ along the line $x_{1}=1$ is easily obtained from Eqs. (8c) and (8a) as

$$
\frac{d \hat{T}}{d x_{2}}=\frac{2 \pi\left(\hat{T}_{c}^{-1 / 2}-\hat{T}_{c}^{1 / 2}\right)}{\left[2 \pi+\left(\hat{T}_{c}^{-1 / 2}-1\right)\left(\pi / 2-\psi_{1}\right)\right]^{2}} \frac{d \psi_{1}}{d x_{2}} .
$$

Because $\psi_{1}$ is continuous in $x_{2}$ and $-\pi / 2 \leq \psi_{1}<\pi / 2$, the denominator on the right-hand side is strictly positive for any $\hat{T}_{c}(>0)$. Thus, it is enough to study $d \psi_{1} / d x_{2}$ to understand the property of $d \hat{T} / d x_{2}$ around $x_{2}=1$. Denoting the $x_{1}$-coordinate of the tangential point by $x_{B}$, the polar angle $\psi_{1}$ of the tangential line is expressed by

$$
\tan \psi_{1}=\frac{-n x_{B}^{2 n-1}}{\sqrt{1-x_{B}^{2 n}}} .
$$

Since the equation of the tangential line from the point $\left(1, x_{2}\right)$ yields the relation

$$
x_{2}=\left(1-x_{B}\right) \tan \psi_{1}+\sqrt{1-x_{B}^{2 n}},
$$



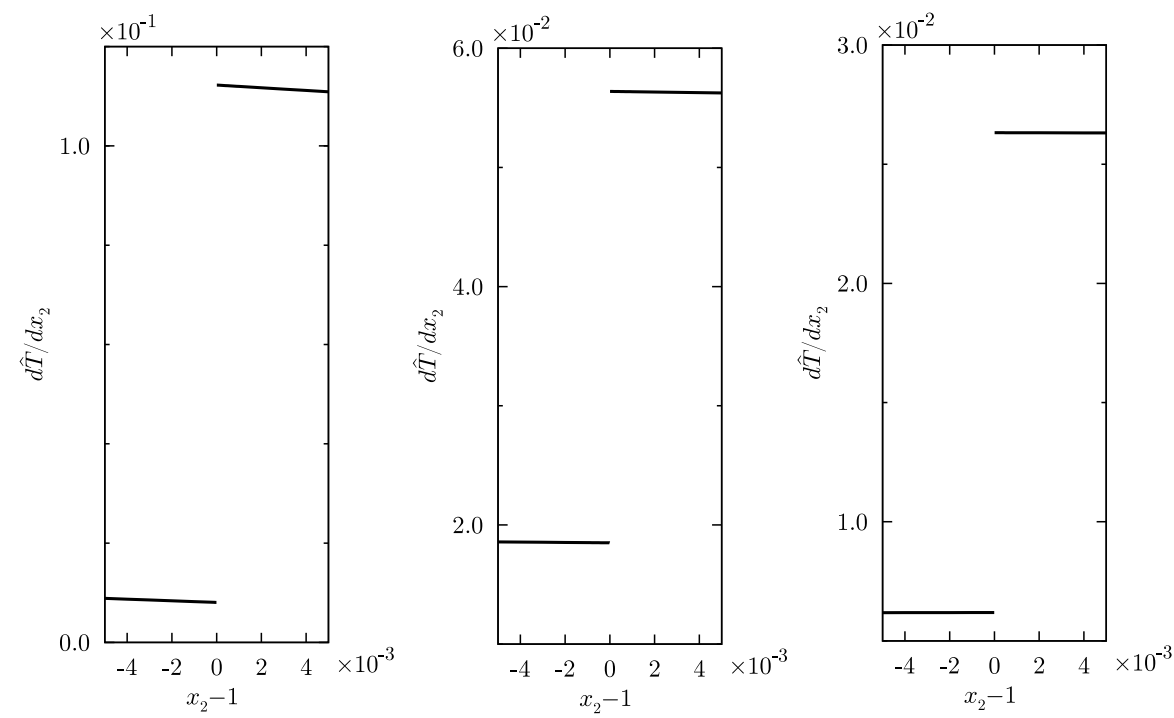

(a) G3
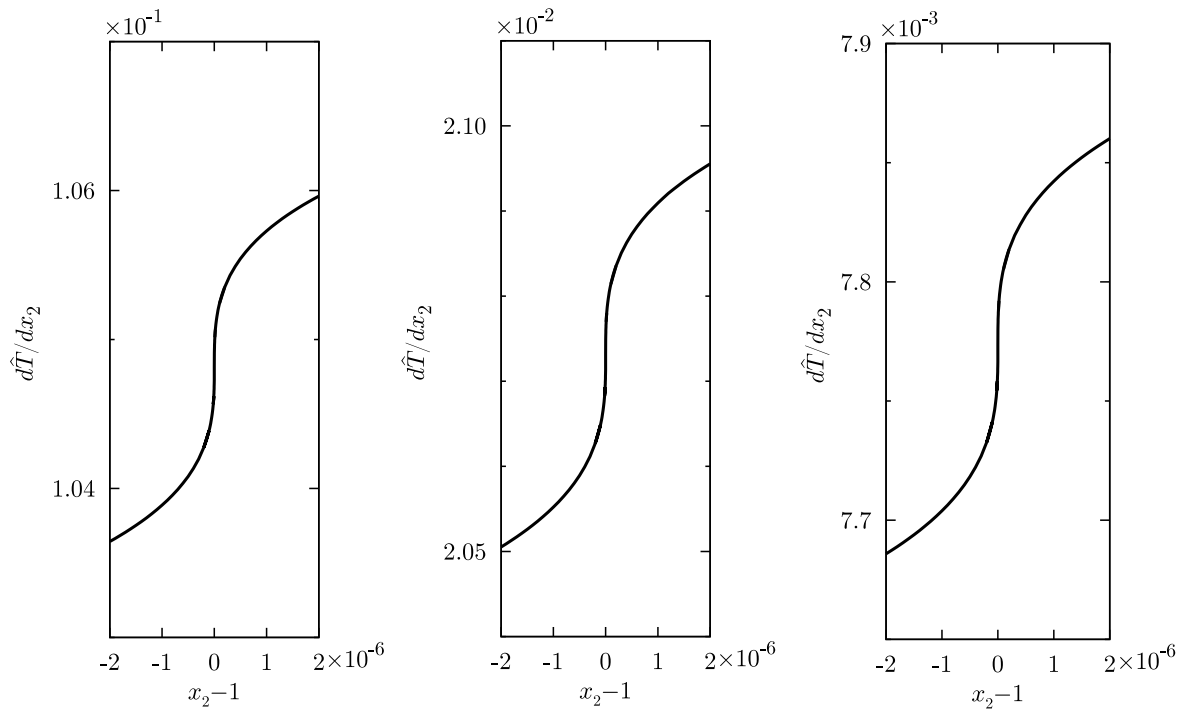

(b) G4

FIG. 10. Temperature gradient in the $x_{2}$-direction along the line $x_{1}=$ const. (a) G3 $\left(\hat{L}=1, \hat{T}_{1}=1, \hat{T}_{2}=2, \hat{T}_{3}=3\right)$. (b) G4 $\left(\hat{T}_{1}=1, \hat{T}_{2}=2\right)$. From left to right, $x_{1}=2,3$, and 4 in (a), while $x_{1}=1,2$, and 3 in (b).

we obtain from Eqs. (14) and (15) that

$$
\frac{d \psi_{1}}{d x_{2}}=\frac{1-x_{B}^{2 n}}{\left(1-x_{B}\right)\left(1-x_{B}^{2 n}+n^{2} x_{B}^{4 n-2}\right)} .
$$

When $x_{2} \simeq 1$, the tangential line with the polar angle $\psi_{1}$ is almost parallel to the $x_{1}$-axis, so that $\left|x_{B}\right| \ll 1$. The relation obtained by eliminating $\tan \psi_{1}$ from Eqs. (14) and (15),

$$
x_{2} \sqrt{1-x_{B}^{2 n}}=1-n x_{B}^{2 n-1}+(n-1) x_{B}^{2 n},
$$

can be simplified for $\left|x_{B}\right| \ll 1$ as $x_{2} \simeq 1-n x_{B}^{2 n-1}$, and we have the estimate

$$
x_{B} \simeq \mp\left|\frac{1-x_{2}}{n}\right|^{\frac{1}{2 n-1}}, \quad\left(x_{2} \gtrless 1\right),
$$




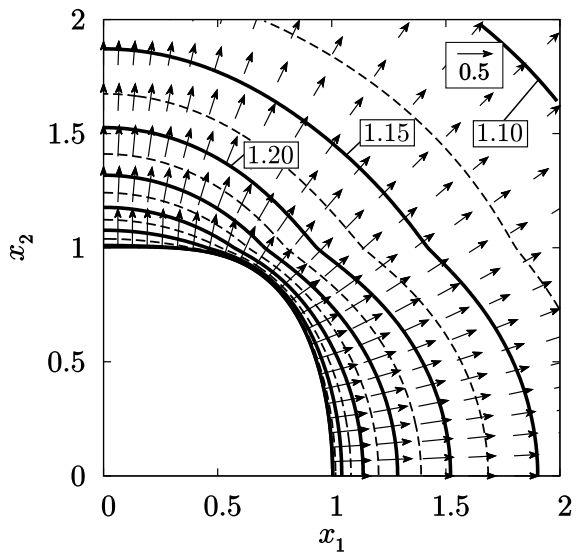

(a)

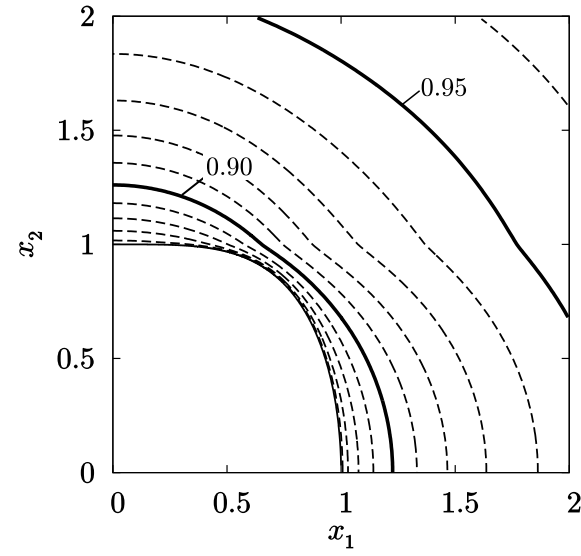

(b)

FIG. 11. Temperature, heat-flow, and density fields for G4 $\left(\hat{T}_{1}=\hat{T}_{2}=2\right)$. (a) The isoline map of $\hat{T}$ and vector field of $\hat{q}_{i}$. (b) The isoline map of $\hat{\rho}$. See the caption of Fig. 4 .

near $x_{2}=1$. Applying this approximation to Eq. (16) finally yields

$$
\frac{d \psi_{1}}{d x_{2}}\left(\simeq 1+x_{B}\right) \simeq 1 \mp\left|\frac{1-x_{2}}{n}\right|^{\frac{1}{2 n-1}}, \quad\left(x_{2} \gtrless 1\right) .
$$

As is already mentioned, the geometry of G4 is recovered by setting $n=2$. Equation (19) in this case tells that $d \hat{T} / d x_{2}$ is continuous but its gradient or its rate of change diverges according to the $-2 / 3$-power law. We refer such a case with diverging second derivative to the "sharp bend." The setting $n=1$ is the case of the circular boundary and gives contrast to the above. Indeed, Eq. (19) with $n=1$ yields the continuous and smooth $d \hat{T} / d x_{2}$, so that the isolines should not have a sharp nor edged bend. This explains the reason why the strange feature of the isolines does not appear in G1 and G2; see Figs. 4 and 5. Incidentally, for any finite $n \geq 2$, Eq. (19) predicts that $d \hat{T} / d x_{2}$ is continuous but not smooth [see $f_{2}$ and $f_{3}$ in Fig. 12(a)]. Therefore, the vanishing or non-vanishing of the curvature of the convex boundary affects the behavior of the isolines on its tangential line. Furthermore, if we take the limit $n \rightarrow \infty$, Eq. (19) is reduced to

$$
\frac{d \psi_{1}}{d x_{2}} \simeq \begin{cases}0, & \left(x_{2}>1\right), \\ 2, & \left(x_{2}<1\right) .\end{cases}
$$

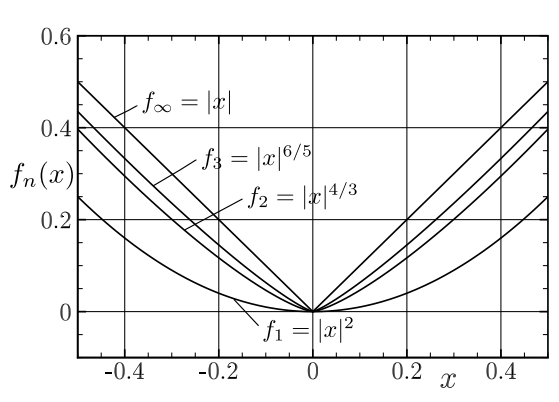

(a)
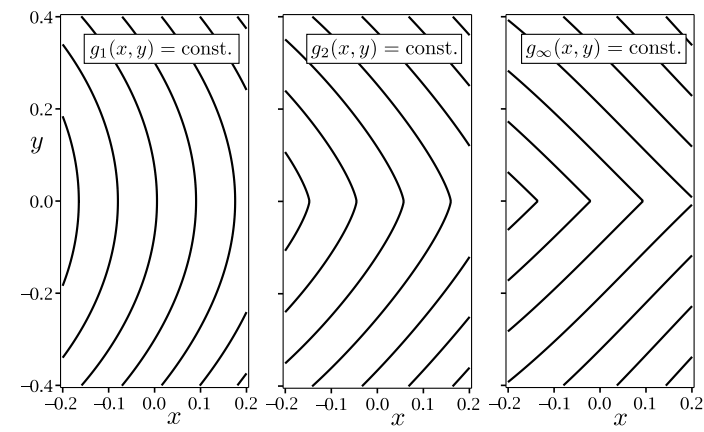

(b)

FIG. 12. Illustration of the smooth, sharp, and edged bends of isolines for the function $g_{n}(x, y)=x+\left(1+x^{2}\right) f_{n}(y)$ with $f_{n}(x)=|x|^{\frac{2 n}{2 n-1}}(n=1,2, \ldots)$. (a) Profiles of $f_{n}$. (b) Isolines of $g_{n}(x, y)$. In (a), $f_{\infty}$ has a jump in its derivative at $x=0$ (edged bend); $f_{3}$ and $f_{2}$ have a continuous first derivative, but their second derivatives diverge at $x=0$ (sharp bend); and $f_{1}$ is smooth. In (b), the isolines show a sharp and edged bend at $y=0$ in $g_{2}$ and $g_{\infty}$, respectively. 
Therefore, $d \hat{T} / d x_{2}$ has a jump discontinuity at $x_{2}=1$. In this way, the edged bend in the isolines in G3 (Fig. 10) is also explained in the same framework. The isolines show a real edged bend on the tangential line to the flat part of the convex body. Figure 12(b) illustrates the feature of the sharp or edged bend of isolines that we discussed.

\section{CONCLUSION}

We have investigated the steady behavior of the free molecular gas in spatially two-dimensional problems, with a special interest in its behavior around a convex body. Four types of setting have been considered as the prototype to draw a general law. We have shown that two types of singularity come out at the level of the macroscopic quantity, even when both of the body-surface temperature and geometry are smooth. Those singularities are due to the propagation of discontinuity of the velocity distribution function, i.e., the effects of the body-surface geometry. We have found a universality and the power-law of the macroscopic quantities near the surface by focusing on its local geometry. Main results are summarized as follows:

1. On the convex body surface, the gradient of macroscopic quantities in its normal direction diverges. This happens at the non-flat part of the body surface. The rate of divergence is determined by the algebraic curve that locally approximates the surface. Especially, at the surface point with nonzero curvature, the rate of divergence is the inverse square root of the distance from the surface.

2. The isolines lose the smoothness in crossing the tangential line at the surface point with zero curvature. In particular, they have an edged bend, if the surface is flat at that point. The isolines remain smooth in crossing the tangential line at the point with nonzero curvature.

When the boundary data, say the surface temperature distribution, cancel out by chance the occurrence of the propagation of discontinuity of the velocity distribution function, the above general law does not apply and no universality would be expected. However, we see that only the regular behavior or a weaker singularity, if exists, occurs, since the dominant contribution to the divergence degenerates.

\section{APPENDIX: ESTIMATE OF THE SECOND TERM OF EQ. (10)}

In this appendix, we show the finiteness or even vanishing of the second term of Eq. (10) as $s \rightarrow 0$.

Because of Eq. (11a), $F^{\prime}$ is written as

$$
F^{\prime}=\frac{d F}{d \theta_{w}} \frac{\partial \theta_{w}}{\partial s} \equiv G\left(\theta_{w}\right) \frac{\partial \theta_{w}}{\partial s} .
$$

Then, the second term of Eq. (10) is rewritten as

$$
\frac{1}{2 \pi} \int_{-\alpha_{*}}^{\alpha_{*}} G\left(\theta_{w}\right) \frac{\sin \alpha}{\sqrt{1-(s+1)^{2} \sin ^{2} \alpha}} d \alpha=\frac{1}{2 \pi} \int_{-\pi / 2}^{\pi / 2} \frac{G\left(\theta_{w s}+\Theta\right)}{s+1} \frac{\sin \beta}{\sqrt{(s+1)^{2}-\sin ^{2} \beta}} d \beta,
$$

where $\alpha=\theta_{w s}-\psi, \alpha_{*}=\psi_{2}-\theta_{w s}=\theta_{w s}-\psi_{1}, \Theta=\theta_{w}-\theta_{w s}$, and $\beta=\Theta+\alpha$. The relative angle $\Theta$ in the argument of $G$ on the right-hand side depends only on $s$ and $\beta$. Moreover, it is odd in $\beta$, i.e., $\Theta(s,-\beta)=-\Theta(s, \beta)$. Using this property, Eq. (A1) is further rewritten as

$$
\text { (A1) }=\frac{1}{2 \pi} \int_{0}^{\pi / 2} \frac{\Delta G}{s+1} \frac{\sin \beta}{\sqrt{(s+1)^{2}-\sin ^{2} \beta}} d \beta,
$$

where $\Delta G \equiv G\left(\theta_{w s}+\Theta\right)-G\left(\theta_{w s}-\Theta\right)$.

When $s$ is small, $\Theta$ is small and thus $\Delta G$ is estimated as $\Delta G \simeq 2\left(d G / d \theta_{w}\right)_{\theta_{w s}} \Theta$. It is also seen from Eq. (11b) that $\Theta \simeq s \tan \alpha=s \sin \beta / \sqrt{(s+1)^{2}-\sin ^{2} \beta}$ for small $s$. We therefore obtain the 


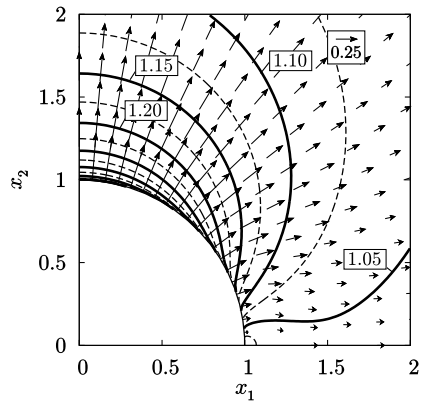

(a)

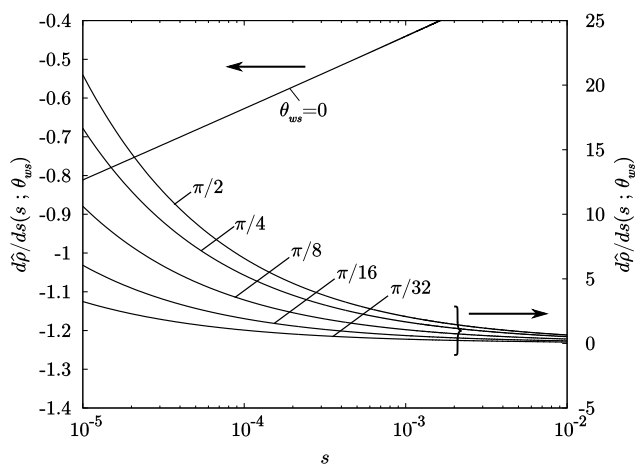

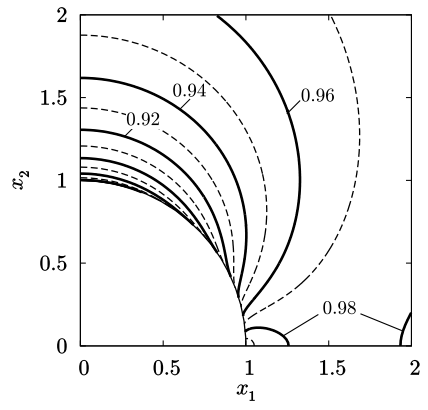

(b)

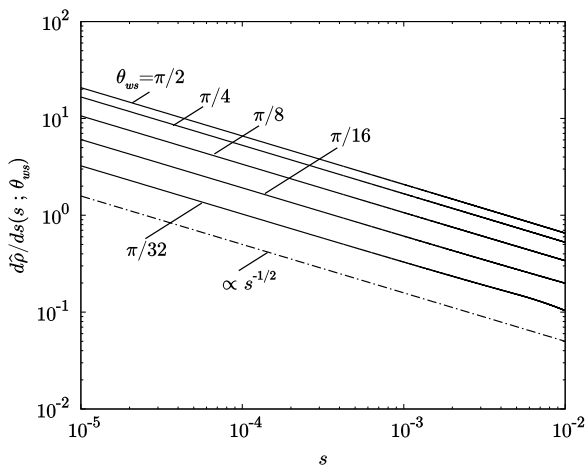

(c)

FIG. 13. Temperature, heat-flow, and density in G1 $\left(\hat{T}_{1}=1\right.$ and $\left.\hat{T}_{2}=2\right)$. (a) The isoline map of $\hat{T}$ and vector field of $\hat{q}_{i}$. (b) The isoline map of $\hat{\rho}$. (c) $d \hat{\rho} / d s$ near the boundary. In (c), the left is the semilog plot, while the right is the log-log plot. See the caption of Fig. 4 for panel (a).

following estimate for the second term of Eq. (10):

$$
\begin{aligned}
(\mathrm{A} 2) & \left.\simeq \frac{1}{\pi} \frac{d G}{d \theta_{w}}\right|_{\theta_{w s}} \int_{0}^{\pi / 2} \frac{s}{s+1} \frac{\sin ^{2} \beta}{(s+1)^{2}-\sin ^{2} \beta} d \beta \\
& =\left.\frac{1}{2} \frac{d G}{d \theta_{w}}\right|_{\theta_{w s}} \frac{s+1-\sqrt{s(s+2)}}{\sqrt{s+2}} \frac{s^{1 / 2}}{1+s}=O\left(s^{1 / 2}\right) .
\end{aligned}
$$

Incidentally, if $\hat{T}_{w}$ is not smooth in $\theta_{w}$ at the surface position $\theta_{w s}, G$ has a jump discontinuity at $\Theta=0$, so that $\Delta G=O(1)$, not of $O(\Theta)$. Consequently, the integral like Eq. (A2) with $\Delta G=1$ remains, from which the divergence with the rate $\ln s$ comes out. This diverging rate is, however, weaker than $s^{-1 / 2}$, so that it dominates the behavior of the gas only when $s^{-1 / 2}$ singularity degenerates, i.e., when $\hat{T}_{w}\left(\theta_{w s}\right)=1$. Numerical results for G1 $\left(\hat{T}_{1}=1, \hat{T}_{2}=2\right)$ supports the present argument, see the case $\theta_{w s}=0$ in Fig. 13(c).

${ }^{1}$ Y. Sone, Molecular Gas Dynamics (Birkhäuser, Boston, MA, 2007); as of January 2016, supplementary notes and errata are available at http://hdl.handle.net/2433/66098.

${ }^{2}$ Y. Sone and S. Takata, "Discontinuity of the velocity distribution function in a rarefied gas around a convex body and the S layer at the bottom of the Knudsen layer," Transp. Theory Stat. Phys. 21, 501 (1992).

${ }^{3}$ Y. Sone, "Highly rarefied gas around a group of bodies with various temperature distributions I. Small temperature variation," J. Méc. Théor. Appl. 3, 315 (1984).

${ }^{4}$ Y. Sone, "Highly rarefied gas around a group of bodies with various temperature distributions II. Arbitrary temperature variation,” J. Méc. Théor. Appl. 4, 1 (1985).

${ }^{5}$ E. H. Kennard, Kinetic Theory of Gases (McGraw-Hill, New York, 1938).

${ }^{6}$ Y. Sone, "Flows induced by temperature fields in a rarefied gas and their ghost effect on the behavior of a gas in the continuum limit," Annu. Rev. Fluid Mech. 32, 779 (2000).

${ }^{7}$ Y. Sone, “Thermal creep in rarefied gas," J. Phys. Soc. Jpn. 21, 1836 (1966).

${ }^{8}$ T. Ohwada, Y. Sone, and K. Aoki, "Numerical analysis of the shear and thermal creep flows of a rarefied gas over a plane wall on the basis of the linearized Boltzmann equation for hard-sphere molecules," Phys. Fluids A 1, 1588 (1989). 
${ }^{9}$ Y. Sone, "A simple demonstration of a rarefied gas flow induced over a plane wall with a temperature gradient," Phys. Fluids A 3, 997 (1991).

${ }^{10}$ Y. Sone, "Flow induced by thermal stress in rarefied gas," Phys. Fluids 15, 1418 (1972).

${ }^{11}$ T. Ohwada and Y. Sone, "Analysis of thermal stress slip flow and negative thermophoresis using the Boltzmann equation for hard-sphere molecules," Eur. J. Mech., B: Fluids 11, 389 (1992).

${ }^{12}$ M. N. Kogan, V. S. Galkin, and O. G. Fridlender, "Stresses produced in gases by temperature and concentration inhomogeneities. New type of free convection," Sov. Phys. Usp. 19, 420 (1976).

${ }^{13}$ K. Aoki, Y. Sone, and N. Masukawa, "A rarefied gas flow induced by a temperature field," in Rarefied Gas Dynamics, edited by J. Harvey and G. Lord (Oxford University Press, Oxford, 1995), pp. 35-41.

${ }^{14}$ Y. Sone and M. Yoshimoto, "Demonstration of a rarefied gas flow induced near the edge of a uniformly heated plate," Phys. Fluids 9, 3530 (1997).

${ }^{15}$ G. Pham-Van-Diep, P. Keeley, E. P. Muntz, and D. P. Weaver, “A micromechanical Knudsen compressor," in Rarefied Gas Dynamics, edited by J. Harvey and G. Lord (Oxford University Press, London, 1995), Vol. I, pp. 715-721.

${ }^{16}$ Y. Sone, Y. Waniguchi, and K. Aoki, "One-way flow of a rarefied gas induced in a channel with a periodic temperature distribution," Phys. Fluids 8, 2227 (1996).

${ }^{17}$ Y. Sone and H. Sugimoto, "Vacuum pump without a moving part and its performance," in Rarefied Gas Dynamics, edited by A. Ketsdever and E. P. Muntz (AIP, New York, 2003), pp. 1041-1048.

${ }^{18}$ H. Sugimoto and Y. Sone, "Vacuum pump without a moving part driven by thermal edge flow," in Rarefied Gas Dynamics, edited by M. Capitelli (AIP, Melville, NY, 2005), pp. 168-173.

${ }^{19}$ S. Takata, H. Sugimoto, and S. Kosuge, "Gas separation by means of the Knudsen compressor," Eur. J. Mech., B: Fluids 26(2), 155 (2007).

${ }^{20}$ C. Cercignani and M. Lampis, "Kinetic models for gas-surface interactions," Transp. Theory Stat. Phys. 1, 101 (1971).

${ }^{21}$ S. Kosuge, K. Aoki, S. Takata, R. Hattori, and D. Sakai, "Steady flows of a highly rarefied gas induced by nonuniform wall temperature," Phys. Fluids 23, 030603 (2011).

${ }^{22}$ S. Takata and H. Funagane, "Singular behaviour of a rarefied gas on a planar boundary," J. Fluid Mech. 717, 30 (2013).

${ }^{23}$ M. Abramowitz and I. Stegun, Handbook of Mathematical Functions with Formulas, Graphs, and Mathematical Tables (Dover, New York, 1972).

${ }^{24}$ P. Welander, "On the temperature jump in a rarefied gas," Ark. Fys. 7, 507 (1954). 